Postgrad. MED. J., (1965), 41, 613

\title{
IMMIGRANTS TO THE UNITED KINGDOM AND VENEREAL INFECTIONS
}

\author{
N. Rosedale, M.R.C.P. (Ed.) \\ Consultant Venereologist, \\ West Middlesex Hospital, Hillingdon Hos pital and \\ Lewisham Group of Hospitals.
}

THE problem of venereal infections in the immigrant population of this country may be summed up as "very much the same as any other inhabitant-but much more often'. Some sexually transmitted infections (lymphogranuloma venereum, chancroid and granuloma inguinale) are more common in the home countries of some of our immigrants. These have always been seen here in small numbers, usually in the region of our seaports, usually in sailors and travellers of all nationalities, and it is surprising that during the years of mass immigration from tropical and sub-tropical countries that there has been no appreciable increase in their reported incidence. For example, the number of cases of lymphogranuloma venereum diagnosed in the venereal disease clinics of England and Wales was 86 in 1955,103 in 1963 and 78 in 1964.

It must be made clear that all figures for the incidence of the various infections dealt with are derived from the annual returns made by the venereal disease clinics of England and Wales to the Ministry of Health. Any infections dealt with by general practitioners or any other hospital department are not recorded. It has been estimated that these unrecorded cases may be in the region of $25 \%$ of the reported cases.

Gonorrhoea is the venereal disease of this country at present, with a reported incidence of 36,049 cases in 1963 (the last full year for which figures have been published). 27,895 of these cases were males. An analysis of 25,942 of these men (by the Co-operative Clinical Group) has shown that of these only $46.3 \%$ were United Kingdom born. No less than $53.7 \%$ of these male cases occurred in foreign born patients $(25.2 \%$ were West Indians, $28.5 \%$ from other countries). (British Co-operative Clinical Group Gonorrhoea Study 1963). Figures 1 and 2 show the marked increase in the reported cases of gonorrhoea over the past few years and the high proportion of foreign born males who contribute to this figure.

\section{Gonorrhoea in the Male}

The amount of gonorrhoea actually brought into this country by immigrants is insignificant; in almost every case the disease is acquired here. It must be accepted that, in general, our immigrants are not of high intelligence and bring the social and sexual customs of their native lands with them. It would also appear that some, West Indians and Mediterraneans in particular, have a highly developed sexual instinct and the women available to them (cheap prostitute or casual pick up) are likely to be infected.

\section{Clinical Picture}

The clinical picture in the vast majority is very straightforward. A heavy purulent urethral discharge is noticed a few days after intercourse, most often in the region of 5 days but incubation periods of 2 to 14 days are acceptable. The patient has, of course, been infectious throughout this period and it is in these days that wives and other regular sexual contacts may be infected. Dysuria may also be noticed but in most cases this is a minor complaint and it is the actual discharge that makes the patient seek medical aid.

It is essential at this stage that the urethral pus is examined bacteriologically by a Gram stained smear and, possibly, by culture. Gonococci are usually seen as the only organism present in the urethral pus and appear as gram negative diplococci.

If the patient has passed urine immediately before examination, pus may be scanty and it is advisable to repeat the examination when more pus has collected before a diagnosis is made and any treatment offered.

When no gonococci can be demonstrated (if necessary, after repeated examination) and when no other obvious cause for the presence of urethral pus can be found e.g. trichomonas vaginalis, the diagnosis, by exclusion, is that of non-gonococcal urethritis. Before any treatment is offered it is advisable to take a sample of blood for Wassermann test, etc. This test at 


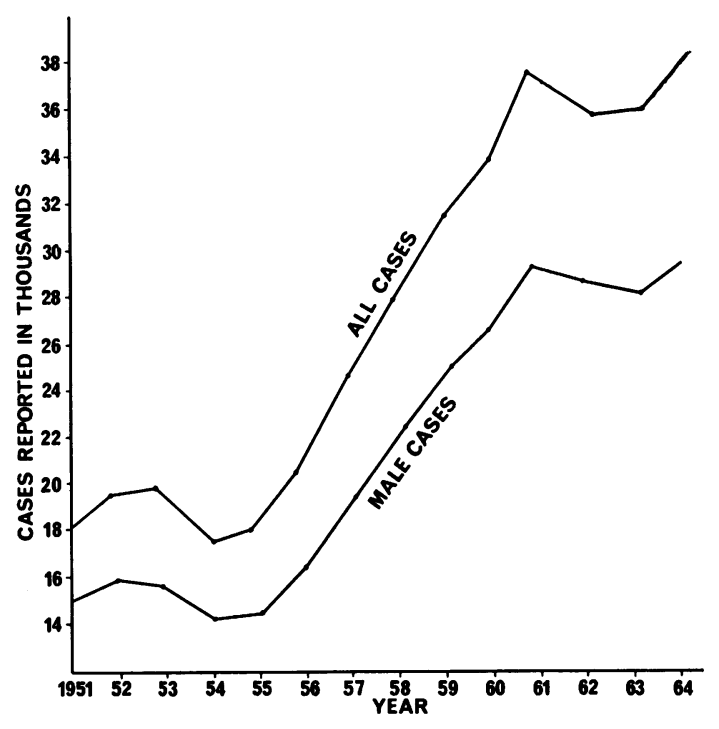

FIG. 1.-Reported incidence of cases of gonorrhoea treated for the first time in clinics of England and Wales 1951-1964.

$\begin{array}{cccc}\text { (From annual reports of Chief } \begin{array}{c}\text { Medical } \\ \text { the Ministry of Health) }\end{array} & \\ \text { Year } & \text { Males } & \text { Females } & \text { Total } \\ 1951 & 14,975 & 3,089 & 18,064 \\ 1952 & 15,510 & 3,585 & 19,095 \\ 1953 & 15,242 & 4,021 & 19,263 \\ 1954 & 13,962 & 3,574 & 17,536 \\ 1955 & 14,079 & 3,766 & 17,845 \\ 1956 & 16,377 & 4,011 & 20,388 \\ 1957 & 19,620 & 4,761 & 24,381 \\ 1958 & 22,398 & 5,489 & 27,887 \\ 1959 & 24,964 & 6,380 & 31,344 \\ 1960 & 26,618 & 7,152 & 33,770 \\ 1961 & 29,519 & 7,588 & 37,107 \\ 1962 & 28,329 & 7,109 & 35,438 \\ 1963 & 27,895 & 8,154 & 36,049 \\ * 1964 & 29,067 & 8,749 & 37,816 \\ & & & \text { (From the 4 } \\ & & & \text { quarterly re- } \\ \text { turns of 1964) }\end{array}$

this time, of course, is of no value in detecting a syphilitic infection acquired at the same time as the gonorrhoea but is necessary as a "base line'.

When gonococci have been found and examination has not revealed any sign of any other infection, treatment may be started at once. The drug of choice is penicillin and a single injection of procaine penicillin in the order of 900,000 to $1,200,000$ units will cure the great majority (in the region of 90 to $95 \%$ ). In cases of penicillin sensitivity, a short course of tetracycline ( 0.25 g. q.d.s. for 4 days) gives almost as good results. A third, and very

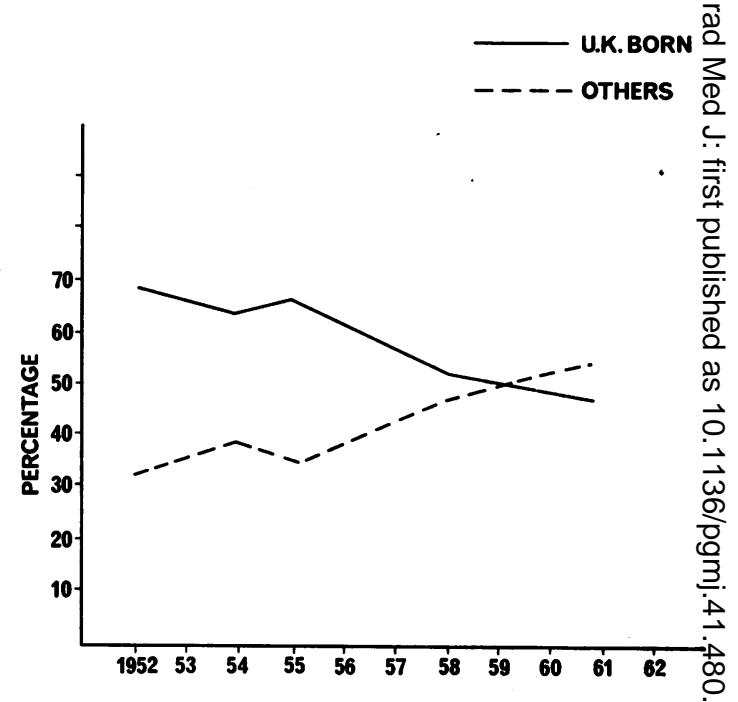

FIG. 2.-Estimated relative numbers of male patients with gonorrhoea 1952-62 (after Willcox, 1965)

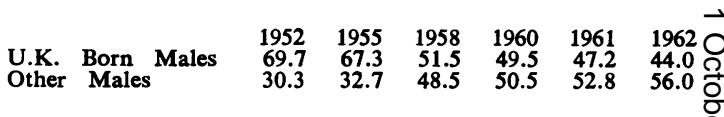

Percentages of U.K. born and other males witi gonorrhoea in co-operative group studies.

efficient, alternative is chloramphenicol ife similar dose.

True penicillin resistance of gonococci is extremely rare but the coincidence of a strain of gonococci with a degree of resistance to the drug and a poorly absorbed penicillin injection may result in a treatment failure. Frequentlye a second injection of a similar dose of penicillin will result in cure. It may be necessary to increase the dose of penicillin (e.g. procaine penicillin 1,200,000 u. daily for 3 days).

Obviously, there will be some exceptions te this very simple account of signs and symptoms diagnosis and treatment of acute gonorrhoeg in the male. It is possible for a gonococcat urethritis to exist with only the most minimad urethral discharge. A scraping of the terminaf urethra with a platinum loop when the patien has not passed urine for some hours (preferabl overnight) should demonstrate the infection?

It is also possible (but very unusual) fore gonorrhoea to be entirely asymptomatic and b\&्छ demonstrable only in the prostatic secretione

\section{Complications}

Under present conditions, complications of acute gonorrhoea in the male are uncommon. Direct spread of the infection into periurethrad tissues may (with secondary organisms) forn 
an abscess. Spread via the posterior urethra to the vas deferens and infection of an epididymis is the most common early complication, usually when the patient has neglected his urethral discharge for some days or weeks.

Late complications should not occur under present conditions when the infection can be diagnosed and adequately treated within a very short space of time. However, some of the older immigrants may have had one or more infections in their own countries that were inadequately treated or not treated at all. Such patients may have a urethral stricture. This may be entirely asymptomatic or cause some loss of power in the urinary stream that the patient has got used to over the years. Some may present as retention of urine when the oedema of a fresh urethral infection blocks a distorted, scarred urethra.

The possibility of a stricture is often suggested by the presence of a slight urethral discharge containing some pus cells and large numbers of secondary organisms even when the specimen is taken with special care from as far within the urethra as the platinum loop (and the patient!) will allow. The gentle passage of a gum elastic bougie or urethroscopy would then be indicated, although such mechanical interference may be postponed until secondary infection is under control.

\section{Follow Up}

Follow up of these cases serves two purposes. Firstly, one establishes that the original infection has been cured by demonstrating the disappearance of urethral pus. When no discharge is obvious, the urine is inspected as a low grade urethral infection will produce pus 'threads' easily seen in a freshly passed specimen.

The other function of the follow up of the treated patient is the exclusion of syphilis (or any other venereal infection) that may have been acquired at the same time as the gonorrhoea. It is usually considered desirable to examine the patient clinically and serologically at monthly intervals for three months after the original infection. There is reason to believe that the treatment of acute gonorrhoea with a large injection of penicillin (e.g. 1,200,000 units of procaine penicillin) will cure an early infection of syphilis that may have been acquired at the same time. It is obviously impossible to diagnose early syphilis in the first few days of its incubation period and, under present conditions, it is still advisable to attempt the full 'traditional' follow up.
In the majority of cases, however, the patient will have had a number of other sexual exposures in the meantime and following the one 'risk' that results in an infection of gonorrhoea and ignoring all the others cannot be considered practical medicine. Encouragement for regular attendance at a clinic for routine serological testing by all promiscuous patients is reasonable.

It must be accepted that a fair proportion of the more irresponsible patients (with many West Indians among them) will not return to the clinic after receiving one injection of penicillin whatever advice or instructions they may be given. Another group will default after their first follow up visit a few days after treatment (or any visit to the clinic when they are not actually given treatment in one form or another). Such patients usually return to the clinic at once if any further signs or symptoms develop and it is difficult to say that any real harm commonly results from such carefree behaviour.

\section{Gonorrhoea in the Female}

The reported number of cases of gonococcal infection in females in 1963 was 8,154. Details of country of origin of 7,459 of these cases show that of these women $77.9 \%$ were born in the United Kingdom, $10.5 \%$ were West Indians and $11.6 \%$ were other immigrants. (British Co-operative Clinical Group Gonorrhoea Study 1963). When the female immigrant is involved in a venereal infection she is frequently a secondary contact i.e. the infection is passed on to her by her husband or other regular sexual partner after he has acquired the infection from an 'extra marital' sexual contact.

\section{Clinical Aspects}

The most important point about a gonococcal infection in the female is that a very high proportion of cases (in the region of $50 \%$ ) are entirely asymptomatic. In others, the symptoms and signs may be very slight and, in themselves, do not encourage an early visit to a doctor or a clinic. Most females with gonococcal infections attend for medical advice only when their male partners have announced their own infections.

A definite history of gonorrhoea in a sexual contact of a female should infer a similar diagnosis in her until it can be confirmed or excluded by repeated bacteriological examination. It is not generally realised that a number of repeated examinations by smear and culture from urethra and cervix (and sometimes, 
rectum) may be necessary to demonstrate the gonococcus in an infected and infectious woman. The number of negative tests necessary to exclude an infection cannot be laid down but must depend on the venereologist's clinical impression and the patient's history including, of course, all possible information on possible infection in her consort or consorts. It is not rare for' the gonococcus to escape detection until six or more serial smears and cultures have been taken.

This is another field of venereology where the writer suggests a practical approach to a practical subject. The gonococcus may be found in an infected female on her first examination; on the other hand it has been stressed that an accurate diagnosis may be delayed for some time. Many female patients will not return to the clinic for repeated examinations whatever is said to them. Many West Indian and Mediterranean males will insist on resuming sexual activity with their women within a day or two without any regard to any medical advice, diagnosis, tests or treatment.

Obviously, a firm diagnosis before any treatment is offered is highly desirable for both doctor and patient, but this ideal requires some measure of co-operation from the patient. In dealing with a fair proportion of immigrant females (or English women who have an immigrant male as their sexual partner) it is an unfortunate fact that any delay in diagnosis or treatment will result in re-infection of the male or a defaulting female until re-infection of the male has occurred. Such delays and defaults also increase the chances of important complications e.g. salpingitis, in the females. Many immigrant females and female consorts of immigrant males have no interest whatever in a diagnosis but simply wish to get rid of any 'germ' that they may have.

One cannot lay down any firm rule of management but offering treatment to a probably infected female is reasonable even when first tests are negative if this is all that the patient wants. It should, of course, be explained to the patient that the giving of treatment implies a diagnosis of gonococcal infection and that routine follow up examinations are necessary.

\section{Treatment}

When a diagnosis has been made or the decision made to treat as a contact of gonorrhoea, treatment is given as for the male patient, a single injection of procaine penicillin
(900,000 units or $1,200,000$ units) being all that is necessary for the majority. Larger dosas are, of course, necessary if there are any complications. Other antibiotics, tetracycline etc. may be necessary.

\section{Follow Up}

The difficulty of demonstrating the gono coccus before treatment in some females has been stressed. It follows that tests of cure a equally unreliable when performed on only one or two occasions after treatment. Here again. some happy compromise between what is clinically ideal and what is acceptable to the patient is necessary. In most cases, if thre serial smears and cultures at weekly intervał after treatment are negative, the patient is probably cured. It is unfortunately true that the best way to diagnose gonorrhoea in female is to have intercourse with her! The majority of female immigrants will have resumed an active sex life long before this $\mathbf{B}$ sanctioned by their medical advisers. Infectio $\overrightarrow{\vec{n}}$ is, of course, not always transmitted by a single act of coitus but, in time, a healthy mas partner (or partners) confirms a cured female

Complications of Gonorrhoea in the Femal

The most important complication of go rhoea in the female is salpingitis, acute subacute or chronic but is not necessarily more common in immigrant women than in any othei socially comparable group.

Diagnosis is best made by demonstrating the gonococcus in specimens from the usual sites (urethra, cervix and possibly rectum) in the presence of salpingitis.

The Gonococcal Complement Fixation Tesł may be of some limited value in this type of case. On the whole, it is an unreliable test with a high incidence of false positive reactions in a low titre. However, the repeated finding of a strongly positive G.C.F.T. in a female with salpingitis suggests that the original causês of her condition may have been gonococcaP. It does not necessarily follow that the present episode of salpingitis is gonococcal. It may be that the original specific infection occurred some time in the past and that secondary infection of a damaged tube accounts for the present flare up of acute or subacute salpingitis

\section{Treatment}

Bed rest is indicated and the usual nursing care of a patient with pelvic inflammation. In the very acute case, where differential diagnosis from acute appendicitis may be difficult 
penicillin $(500,000$ units of crystalline penicillin 6 hourly for a few days) is often dramatically effective. In the more commonly seen subacute and chronic cases, bed rest is always indicated too but broad spectrum antibiotics may be more effective than penicillin. When possible, antibiotic therapy should be related to culture and sensitivity of organisms in a cervical swab. Obviously, more direct bacteriological examination of the infected Fallopian tube is impossible.

Flare up of a subacute-on-chronic salpingitis is, unfortunately, not uncommon and in some cases removal of the damaged tube (tubes) is indicated as the only alternative to frequent episodes of ill health, dyspareunia, etc.

Other complications, abscess of the duct of Bartholin's gland, Skene's abscess and the very rare gonococcal arthritis are dealt with by removing the focus of gonococcal infection with adequate doses of penicillin. Otherwise, basic surgical principles apply and pus, once formed, must be drained. Bartholin's abscesses require adequate incision and drainage when they do not often reoccur. Frequent recurrence may be an indication for surgical removal of the gland and its duct.

\section{Syphilis}

The incidence of early infectious syphilis (primary, secondary and latent in the first year of infection) treated in the venereal disease clinics of England and Wales in 1963 was 1,390 (Males 1,135, Females 255). An analysis of the racial groups making up this figure suggest that the male numbers were made up of $62.6 \%$ U.K. born, $6.6 \%$ West Indians and $30.7 \%$ other immigrants. The figures for women were $59.4 \%$ U.K. born, $29.1 \%$ West Indians and $11.5 \%$ other immigrants. (Annual Report of Chief Medical Officer of Ministry of Health 1963.) This distribution is markedly different from the distribution of gonorrhoea.

The longer incubation period of syphilis makes it a disease more likely to be brought into the country by immigrants.

An attempt is made by Health Officers to diagnose early infection by examination on arrival at Ports of entry, and, when a diagnosis of early infectious syphilis is confirmed, the immigrant is refused admission to the country. The numbers dealt with in this way must be minute.

The clinical details of the various stages of syphilis are beyond the scope of this paper. Basic principles apply and any genital ulceration should suggest at least the possibility of an early syphilitic infection. Investigation by dark ground microscopy of serum from any genital ulcer for Treponema pallidum and by serial blood tests are necessary to exclude the infection: the former continuing as long as an open ulcer is present, the latter until at least three months has elapsed after the last possible date of infection.

Latent Syphilis is a diagnosis of exclusion. Standard serological tests (the Wassermann Reaction, Kahn test, V.D.R.I., Price's Precipitation Reaction, etc.) are confirmed as positive on repeated testing: it is usual to confirm these results with one or more of the more specific tests (Reiter Protein Complement Fixation Test, Fluorescent Treponemal Antibody Test or Treponemal Immobilisation Test).

Primary and secondary syphilis are excluded by negative clinical examination. Late syphilis, particularly lesions of the cardio-vascular and nervous systems are excluded, again by general physical examination, chest X-ray and screening for cardiac and aortic contours and by examination of the cerebro-spinal fluid by lumbar puncture. The important CSF investigations are cell count, protein content, globulin estimation, Lange curve and Wassermann reaction.

A diagnosis of latent syphilis is, of course, an indication for treatment. When any manifestation of late syphilis is discovered other general medical care and treatment may be necessary in addition to a course of antisyphilitic treatment.

A practical point in the management of a patient with early infectious syphilis is the matter of defaulting by the more irresponsible (not necessarily an immigrant). It is often advisable to give a large initial dose of long acting penicillin (e.g. 2,400,000 units of benzathine penicillin, given in two injections, one into each buttock) on diagnosis of primary or secondary syphilis. Many of these cases will be cured by this dose even if they never return for further treatment. The course of treatment for the vast majority who attend as requested involves a course of procaine penicillin amounting to a total dose in the region of $10 \mathrm{mega}$ units. A suggested routine course, using procaine penicillin with $2 \%$ aluminium monostearate, is an injection of $1,200,000$ units three times weekly to a total of 10 injections.

It should also be remembered that a diagnosis of syphilis at any stage implies the responsibility of arranging examination of sexual contacts and, when indicated, children of patients.

The matter of the diagnosis, treatment and general management of syphilis has not been 
dealt with in detail as the incidence of syphilis in immigrants is not so high as that of gonorrhoea.

\section{Non-Syphilitic Positive Serological Tests}

In the majority of Europeans, a series of repeated strongly positive serological tests for syphilis suggests past or present infection with Treponema pallidum. This may have been treated, inadequately treated or untreated. Biological false positive reactions may occur and have been attributed to a variety of acute and chronic infections. In many cases, no obvious reason can be found for the false positive reaction once syphilis has been excluded as the cause mainly by the more specific tests previously mentioned (R.P.C.F.T., F.T.A., T.P.I.).

All this applies equally to the immigrant patient but, in this group, the non venereal treponematoses have also to be considered.

\section{Treponematoses}

There are various diseases found in different parts of the world that are due to organisms similar to the Treponema pallidum of syphilis. Whether these are closely related infections or the same infection modified by time, social customs, etc. is not certain. There are various theories including the suggestion (again under change of environment, etc.) that venereal syphilis can change to the non-venereal treponematosis and vice-versa. One of the treponematoses, yaws, which is endemic in the tropical and sub-tropical home countries of so many of our immigrants is our main concern in this country.

The causative organism of yaws is the Treponema pertenue, and is indistinguishable from the Treponema pallidum. The relationship between the two diseases has been mentioned but will not be gone into further. The important fact is that yaws is a transmissible infection but most often as a result of contact among children and not sexual contact among adults. The primary lesion of yaws may be found anywhere on the surface of the body and the disease may be seen in its early infectious stage in recently arrived child immigrants. The clinical course is roughly comparable to that of syphilis and soon reaches a latent stage or results in cure but possibly leaving the serological tests positive. In the vast majority of cases of immigrants with suspected yaws (treated or untreated) the problem will be one of interpretation of serological tests. Many immigrant patients give a history of yaws diagnosed and treated i childhood; many others have been exposes to the infection but have never been so. diagnosed.

So far as we are concerned, when early lesion have healed there is no clinical or serologica differentiation between the two conditions. A $\overline{\bar{p}}$ adult patient may have been treated for yaw in childhood and then acquired syphilis lates in life.

A suggested procedure to follow wher serological tests of an immigrant from a yaws area (West Indies, Africa, etc.) are found to be repeatedly positive and when a biological false positive has been excluded is:

(1) A careful history of possible yaws infection in childhood, treated or uns treated. The possibility of yaws lesions in brothers and sisters or playmates? describing the typical lesions on the legs is worth enquiring after. A history of venereal contacts, infections, blood tests, penicillin or other antibiotic therapy foe any reason should be noted.

(2) Complete clinical examination to exclude $\mathrm{e}^{\mathrm{C}}$ the lesions of primary or secondery syphilis and the late lesions of cardsour vascular and neuro-syphilis (as previously detailed). At the same time a searct is made for any scars on the legs that have the typical appearance of old yaws lesions. Differentiation of the roughly circular 'tissue paper' scar of the yaws lesion from scars of old trauma anc sepsis may however be difficult of impossible. The scarring and thickening of the skin of the soles of the feet of crab yaws is another suggestive sign? It must be emphasised again that definite history of a yaws infection witl. undoubted signs of old lesions mass coincide with a superinfection with venereal syphilis.

(3) The examination of all available sexuab contacts and, where indicated, children? in an attempt to find evidence of syphilisn In practice, this procedure is not of very great value. There have probablis been many other sexual contacts besidess the one(s) immediately available and the contacts may also have had yawse

(4) The titre of the positive serological test may be an additional indicator, very weak positives in young patients sug gesting yaws. Strong positive reaction $\bar{s}^{\circ}$ in older patients suggest syphilis buf 
unfortunately are of very little real diagnostic value.

(5) Treatment. As there is no means of absolute differentiation between the venereal and non-venereal treponematoses, the only practical step is to offer a course of treatment sufficient to cure syphilis if it is present. The course may be as previously suggested or may be modified (to suit the patient) to injections of benzathine penicillin in larger doses at greater intervals (e.g. Penidural L.A. 2,400,000 units $x 3$ doses at intervals of 10 days to two weeks).

(6) Serological and clinical follow up of the patient as in a case of treated syphilis.

\section{Pregnant Women}

The one group in which this problem is seen particularly frequently is the pregnant immigrant female. Their fertility rate appears to be high and the routine blood test performed in the course of each pregnancy yields a high proportion of positive reactions. The routine procedures enumerated above must be gone through on each occasion. In the new case, a history of one or many previous healthy pregnancies in their home country suggests that the cause may not be syphilitic but there is no alternative to offering a standard course of treatment.

Even in the case of a woman previously examined and treated during one or many pregnancies in this country, the presence of persistently positive serological tests is usually an indication for a further course of penicillin as one can never be absolutely certain that syphilis has not been acquired between pregnancies. Examination of sexual contacts each time is of value; if the consort remains the same and he is sero-negative on each occasion, recent syphilis in the female is that much more unlikely.

The generally accepted practice in most of the clinics of this country is that such pregnant immigrant women are offered a further course of penicillin during each pregnancy while their serological tests remain positive to avoid (however rarely it might happen) the possibility of a syphilitic foetus.

Penicillin sensitivity is a possible danger. When it exists or develops during treatment, drugs of the tetracycline group may be substituted. There is evidence that these drugs can damage the foetus if given in the last three months of pregnancy. In such a case, the question of whether or not to treat would require careful consideration and a decision for each individual case. As in all treated syphilitic women the child is observed at regular intervals after birth. If it remains well in all respects, a clinical examination and blood tests at the age of about 6 weeks (when the 'carry over' of maternal reagin to the infant's serum has probably disappeared) are desirable to confirm a healthy child.

\section{The 'Tropical' Venereal Diseases}

These are placed last because, although the most specific venereal diseases of the immigrant population, they are numerically almost insignificant when compared to the immigrants' share of the standard venereal diseases of this country-gonorrhoea and syphilis.

\section{Lymphogranuloma Venereum}

In the case of this infection it is quite likely that the reported incidence as shown in Table does not represent the true figure. The later manifestations in older patients are more likely to be dealt with in other hospital departments and never referred to the Venereal Disease Clinic.

Lymphogranuloma venereum is a virus infection and is usually transmitted by sexual contact. The primary lesion appears on the genitalia within a few days of contact and usually takes the form of a small papule or herpetiform vesicle. It is frequently unnoticed by the patient. The usual presenting signs and symptoms develop later (usually 3 to 5 weeks later) and takes the form of pain and swelling in one or both groups of inguinal glands. Clinically, this is recognised as an adenitis and periadenitis. The infection is, as a rule, subacute and (in a white-skinned patient) the inflamed area has a bluish tinge very characteristic of this condition. Suppuration usually develops and, if neglected, may discharge through the skin. Some cases may present as acute relapses of a subacute infection: in these, chronic induration of the inguinal area and signs of partly healed or healed sinuses may be found. The lesions are not usually so tender that bending the leg is impossible and the swelling in the groin may show the skin fold of movement of the hip (this is a point in the differential diagnosis from the buboe of chancroid or other more acute septic adenitis).

Diagnosis. The Frei Test is of some value in confirming the diagnosis of L.G.V. This is an intradermal injection of killed virus, usually into the skin of the forearm with a control injection of the suspending fluid into the skin 
TABLE 1

RePORTED InCIDENCE OF ChanCroid, Lymphogranuloma Venereum and Granuloma Inguinale in the Venereal Disease Clinics of ENGLand and WALES (1957-1964).

\begin{tabular}{ccc} 
Year & \multicolumn{2}{c}{ Chancroid } \\
& Male & Female \\
1957 & 254 & 6 \\
1958 & 247 & 12 \\
1959 & 265 & 2 \\
1960 & 226 & 5 \\
1961 & 227 & 1 \\
1962 & 154 & 3 \\
1963 & 153 & 6 \\
$* 1964$ & 136 & 4
\end{tabular}

Lymphogranuloma Venereum

Granuloma Inguinale

76
77
80
102
98
96
103
78

*1964 figures estimated from the four quarterly returns of 1964

of the other arm. A papular reaction with an area of indurated erythema at the site of injection (usually read at 48 hours) is recorded as a positive reaction. The disadvantages of this test are that it is sometimes delayed even in the presence of definite early lesions of L.G.V. (Erskine, 1958) and that a proportion of immigrants produce positive Frei reactions without clinical evidence of the infection. King, Barwell and Catterall (1956) confirmed the diagnosis in only $2 \%$ of a total group of 1,119 patients (991 white, 118 coloured) when the total of positive tests was $18.4 \%$. This study was performed in the earlier days of mass coloured immigration and their findings in the two groups was: White positive reactors, male $16 \%$, female $11 \%$, Coloured positive reactors, male $50 \%$, female $36 \%$.

The Complement Fixation Test is a little more sensitive as a diagnostic test. It is generally accepted that a titre of 1.32 or more is necessary to establish the diagnosis. In early cases, it is of value to demonstrate a rising titre of the L.G.V. Complement Fixation Test in specimens examined at intervals of (say) two weeks. Both the intradermal test and complement fixation test will, of course, produce positive reactions in the presence of infection with other viruses in the psittacosis-lymphogranuloma group.

Treatment. Local. Once pus has formed in a buboe, it should be aspirated using a wide bore needle. Allowing spontaneous rupture or incision frequently results in the development of a chronic sinus.

General. The present position and opinions on the value of antibiotic therapy in the acute condition has been summarised in Recent Advances in Venereology (King, 1964). Oxytetracycline seems to be the most satisfactory drug in doses of the order of $2 \mathrm{~g}$. daily ove a period of about 21 days. Alternating this course with sulphonamides in doses of 4 g daily is another suggested regime.

The Ano-Rectal Syndrome of Lymphogranuloma Venereum is not uncommon, but is seen more frequently in hospital departmen 8 other than the venereological dept. It is seegr more often in females and it has been suggested (Grace, 1943) that most cases of proctitis fond rectal stricture in males result from hom sexual contact. In the majority of his cases, $(73.8 \%$ of 145 cases) the clinical picture was stricture with proctitis. In the older immigran female, stricture without proctitis may be the late result of genital infection with spreag (with suppuration, scarring etc.) to the para rectal lymph glands.

Treatment is along the lines described for the inguinal or genital form. Mechanical dilatatic of rectal strictures may be necessary. Somes times, more drastic surgery with temporary of permanent colostomy may be indicated. Lym phogranuloma venereum should certainly be considered in the diagnosis of proctitis and rectal stricture in an immigrant patient.

\section{Chancroid.}

This is a specific genital infection caused by Haemophilus ducreyi. It is see commonly in tropical and sub-tropical regions of the world and is associated with lack of hygiene. The incidence in this country is now high (Table 1).

The incubation period is usually only matter of 2 to 4 days. The disease presents as one or more superficial, painful ulces with ragged undermined edges. These lesions may soon be associated with involvement of the inguinal lymph glands resulting in very painf 


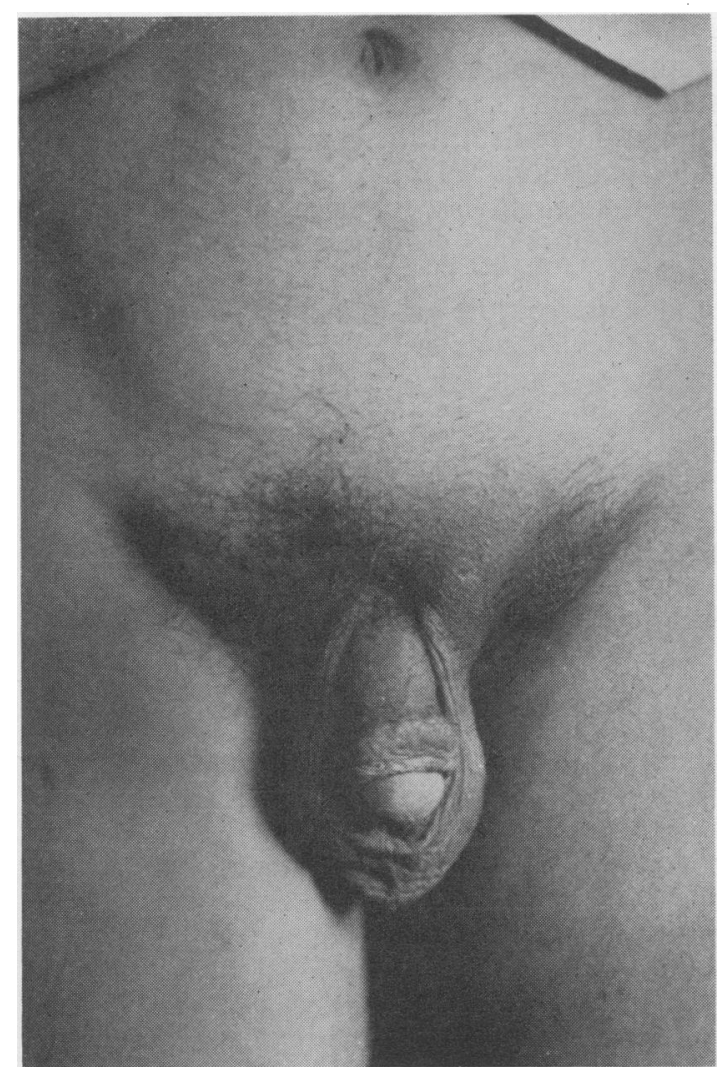

FIG. 3.-Bilateral inguinal buboes of lymphogranuloma venereum.

ful adenitis and periadenitis. This, as a rule, is much more acute than the buboe of lymphogranuloma venereum. Spontaneous rupture may occur shortly after the appearance of suppuration.

Diagnosis may be made by demonstrating the causative organism but this is not easy. Ducrey's bacillus does not grow well in the presence of other organisms and specimens from open, secondarily infected, ulcers are not satisfactory. The best material is obtained by aspiration of an unruptured buboe.

Intradermal Test. The Ito-Reenstierna test, the intradermal injection of a killed suspension of Ducrey's bacillus is of limited use. A high incidence of positive reactions has been reported in apparently normal individuals (Willcox, 1952) and although these may be true reactions indicating contact with the organism at some time, the test is not of great value in the immediate diagnosis of a genital ulcer.

Diagnosis by biopsy is one suggested procedure (Heyman, Beeson and Sheldon, 1945) but is often not a practicable procedure, mainly from the patients' point of view!
To summarise: the diagnosis of chancroid as the cause of genital ulceration is most often made on clinical grounds (short incubation period followed by the development of multiple painful ragged ulcers and associated inguinal buboe(s)). Isolation of the causative organism by smear and culture is best attempted in material removed from an unruptured buboe by aspiration. Perhaps a more important duty is the exclusion of a syphilitic infection (by the usual procedures of dark ground examination of material from the ulceration and serial blood tests). Fortunately, chancroidal infections will respond to drugs which are not treponemicidal so that treatment of chancroid and exclusion of syphilis can proceed together.

Treatment. Sulphonamides are effective. $1 \mathrm{~g}$. four times daily for 10 to 14 days is often all that is required.

Streptomycin, $1 \mathrm{~g}$. daily for five days has produced good results (Asin, 1952). A combined course of streptomycin and sulphonamides is perhaps the most effective of all.

Other antibiotics, the tetracyclines and chloramphenicol are also effective but are best avoided as they will interfere with routine testing to eliminate syphilis (in this country, very much more common as a cause of genital ulceration than chancroid).

\section{Granuloma Inguinale}

This must be considered in the differential diagnosis of genital ulceration in an immigrant patient. As Table 1 shows, the reported incidence in this country is still minute, only 10 cases in 1963 and the figure for 1964 is likely to be even smaller ( 7 cases reported so far, in the four quarterly returns for 1964).

Granuloma inguinale is caused by the Donovan body (Donovania Granulomatis) and may be demonstrated by smear or biopsy of the lesion.

This condition is generally considered with the sexually transmitted conditions but, since the condition is rarely seen in both sexual partners, its classification as a venereal disease is in doubt. Greenblatt (1958) (quoted by Barrow, 1958), in a series of several hundred patients with granuloma inguinale in Georgia, U.S.A., found only about 6 cases of conjugal infection.

Clinical Appearance. Single or multiple granulomatous ulcers on or close to the genitalia with a smooth velvet-like appearance is generally described. Differential diagnosis from a malignant ulcer is usually suggested by the naked eye appearance and, in fact, car- 


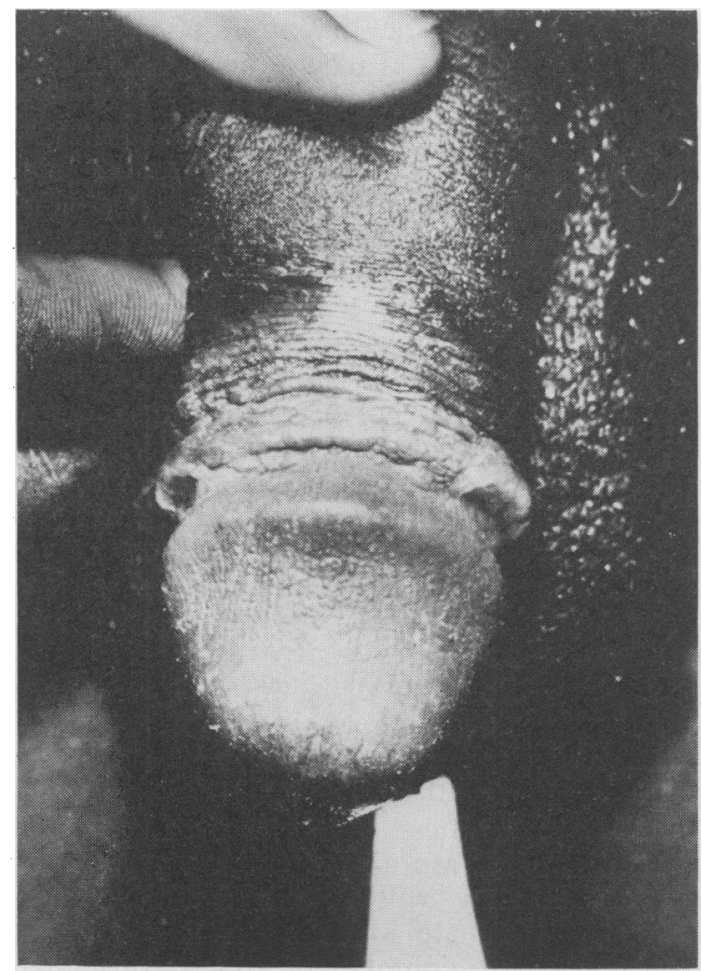

Fig. 4.-Penile lesions of granuloma inguinale

cinomatous change has been reported occasionally.

\section{Treatment}

Streptomycin is the drug of choice. $4 \mathrm{~g}$. daily for five days has been suggested (Greenblatt, Barfield, Dienst, West and Zises, 1952).

Chloramphenicol and the tetracyclines have also been reported as producing good results, but, again, these are best avoided until syphilis has been excluded as a cause or as a coincidental infection.

\section{Other Sexually Transmitted Conditions}

Non-gonococcal urethritis in males is a condition that has shown an increased incidence over recent years, comparable to the increased incidence of gonorrhoea. No large scale surveys of the country of origin of patients has been undertaken but it would appear that the male immigrant to this country is contributing his disproportionate share of this infection also. Willcox (1965) reporting on 800 consecutive males with non-gonococcal urethritis treated at St. Mary's Hospital, Paddington, during 1961 found that only $40 \%$ were United Kingdom born. Of the $60 \%$ foreign born, $30 \%$ were West Indians, 30\% other immigrants.
It is worth repeating that non-gonococcas urethritis in males is a diagnosis of exclusion- $-\mathbb{2}$ mainly from a gonococcal infection. In the immigrant patient, other causes deserve a little more consideration: Urethritis due to Trichow monas Vaginalis is reported in various serie from $1 \%$ to $15 \%$ of all cases of non-gonococca urethritis. There is no doubt that the more if is looked for, the more it will be found and that the incidence will be markedly higher in the male sexual partners of women found to harbour the parasite in the vagina (to the exten? of $40 \%$ to $60 \%$ of those examined.)

One other cause of urethral pus (often as sociated with a variety of secondary organisms has been previously mentioned: a urethra stricture resulting from one or many previous episodes of urethral infection, untreated or in $\rightarrow$ adequately treated.

Non-Gonococcal Infection in the Female. No's comparable condition to N.G.U. in the malew has been discovered in the female. In ous present state of ignorance, the best that one can do is to examine the female partner when possible and attempt to treat any abnormality that may be found. It has been suggested that mere empirical treatment of the female partiger serves no useful purpose (Rosedale, 1958) Also, in the author's experience, many male patients return to the same female partner af treatment of an episode of N.G.U. when thas female has not been examined or treated foํㅡㅁ anything and never suffer a recurrence of theio urethral inflammation.

\section{Summary}

Gonorrhoea and syphilis are the only com monly seen venereal diseases of this country These and other sexually transmitted conditions e.g. non-gonococcal urethritis in the male, areseen very much more frequently than the othez. venereal diseases of the countries of origin of many of our immigrants (chancroid, lympho-3 granuloma venereum, granuloma inguinale).

Immigrants, particularly the males, accoun for a very large proportion of the reported venereal disease of this country (over $50 \%$ of: all new cases of gonorrhoea in males occurringo in foreign-born men).

The one specific problem in venereology peculiar to the immigrant alone is the interpreta tion of positive serological tests for syphilis most often found on routine ante-natal blood testing. The differentiation between venereal syphilis and a non-venereal treponematosis as the cause is usually impossible and the usuab practical solution is to treat as for syphilis. 
The 'tropical' venereal diseases must always be considered in the investigation of immigrants with genital and other lesions, particularly those newly arrived in this country, but the vast majority share and spread the diseases that have always been endemic in the normal population of this country.

\section{REFERENCES}

AnNual Report of the Chief Medical Officer of HEALTH FOR THE YEAR 1963, 65, London: H.M.S.O. AsIN, J. (1952): Chancroid. A report of 1,402 cases, Amer. J. Syph., 36, 483.

BRITISH Co-OPERATIVE Clinical Group GonorRHoEa STUDY 1963 (1965): Brit. J. vener. Dis., 41, 24.

ERKSKINE, D. (1958): Lymphogranuloma Venereum. A Review of 61 cases, Brit. J. vener. Dis., 34, 163.

GRACE, A. W. (1943): Anorectal Lymphogranuloma Venereum, J. Amer. med. Ass., 122, 74.

GREenBLATT, R. B., BARFIELD, W. E., DIENST, R. B., WEST, R. M., and ZISES, M. (i952): A Five Year
Study of Antibiotics in the Treatment of Granuloma Inguinale, Amer. J. Syph., 36, 186.

GreENBLATT, R. B., (1958) quoted by BARROW, J. (1958): Granuloma Inguinale in a Jamaican and his Wife, Brit. J. vener. Dis., 34, 34.

Heyman, A., Beeson, P. B., and Sheldon, W. H. (1945). Diagnosis of Chancroid. The Relative Efficiency of Biopsies, Cultures, Smears, Autoinnoculations and Skin Tests, J. Amer. med. Ass., 129, 935.

King, A. J., Barwell, C. F., and Catterall, R. D. (1956): Intradermal Tests in the Diagnosis of Lymphogranuloma Venereum, Brit. J. vener. Dis., 32, 209.

KiNG, A. J. (1964): Recent Advances in Venereology. p. 320-331, London: J. \& A. Churchill.

RosedAle, N. (1959): Female Consorts of Men with Nion-Gonococcal Urethritis, Brit. J. vener. Dis., 35, 245.

Willcox, R. R. (1952): The Ito Skin Test for Chancroid in the Adult and Juvenile African, Amer. J. Syph., 36, 284.

WILLCOX, R. R. (1965): Immigration and Venereal Disease in Great Britain, Brit. J. clin. Pract. In Press. 